\section{Isenthalpic Flow, Joule-Kelvin Coefficients and Mantle Convection}

WALDBAUM $^{1}$ has suggested that, when mantle material convects according to the model of Turcotte and $\mathrm{Oxburgh}^{2}$, the temperature of an isolated rising mass will increase, "even in the idealized case where friction, viscosity and turbulence are ignored". He argues from the premise that the steady adiabatic flow of a fluid is isenthalpic along streamlines: the JouleKelvin coefficient of the fluid can therefore be used to predict the temperature change during decompression.

This argument is incorrect. Waldbaum has apparently been misled by variations in the published definitions of enthalpy. Although the enthalpy of a substance is always defined by an expression of the form

$$
h=u+p v
$$

( $h=$ specific enthalpy, $u=$ specific internal energy, $p=$ pressure and $v=$ specific volume), there are important variations in the definition of the internal energy $u$. Of the two authorities quoted by Waldbaum, Pippard ${ }^{3}$ includes in $h$ and $u$ terms for kinetic and potential (gravitational) energy, whereas Liepmann and Roshko ${ }^{4}$ include a term for kinetic energy in what they call "total enthalpy", but make no mention of potential energy. For them and for Landau and Lifshit $z^{5}$, however, the unqualified term enthalpy is taken to describe an intrinsic property of a substance, dependent only on local thermodynamic variables; it is "the enthalpy which would be measured by an 'observer' moving with the fluid"4. Pippard's definition permits him legitimately to say that, in steady adiabatic flow, the enthalpy of a fluid is constant along streamlines. For Landau and Lifshitz, however, the quantity which is constant is the sum

$$
\text { enthalpy + potential energy + kinetic energy }
$$

It can now be seen why the Joule-Kelvin coefficient $(\partial T / \partial p)_{h}$ is not directly applicable to free convection in a gravitational field. This coefficient is defined physically for a condition of constant intrinsic enthalpy, which does not exist where decompression occurs through upward movement in a gravitational field. For a slowly moving frictionless fluid, the stress field in those circumstances must be purely hydrostatic, and in adiabatic flow the entropy $(s)$ will be constant. Differentiating (1) and (2) gives

$$
\mathrm{d} u+p \mathrm{~d} v+v \mathrm{~d} p+g \mathrm{~d} z=0
$$

where $g$ is gravitational acceleration and $z$ is vertical height. As $v \mathrm{~d} p=-g \mathrm{~d} z$ in hydrostatic conditions, we obtain the standard equation of energy balance for reversible adiabatic expansion

$$
(\mathrm{d} u+p \mathrm{~d} v)_{s}=0
$$

The temperature gradient is therefore the well known adiabatic gradient of geophysical fluid dynamics, based on the isentropic coefficient $(\partial T / \partial p)_{s}$.

It might be suggested that isenthalpic heating or cooling coefficients could be applied to deviations of the stress field in a real fluid from a vertical hydrostatic gradient. Such deviations could be considered to correspond to the irreversible "isenthalpic steps" of Walbaum's argument. But how large are they? The topography of the mid-ocean rift suggests $\sim 0.6$ kbar near the surface, whereas Mackenzie ${ }^{6}$ obtained values less than $1 \mathrm{kbar}$ at depths of 50 to $100 \mathrm{~km}$ under trenches from an analysis of gravity anomalies. Waldbaum's isenthalpic coefficients therefore suggest temperature rises of $\sim 20 \mathrm{~K}$ or less. It is also questionable whether the flow is truly adiabatic in the regions of maximum non-hydrostatic stress; as $\operatorname{Tozer}^{7}$ points out, when viscosity of a fluid varies rapidly with temperature, shear flow and associated viscous dissipation will tend to be strongly localized. The evidence of high heat flow behind island arcs tends to support this view ${ }^{8}$. Thus it seems likely that Joule-Kelvin coefficients will be of limited value in estimating temperature changes related to slow planetary convection.

Because of these problems it seemed worthwhile to examine further the variations in usage of the concept of internal energy among thermodynamicists. Many workers bypass the problem by not mentioning gravitational and kinetic energy at all. Gibbs ${ }^{9}$ clearly separated gravitational energy from what he called the "intrinsic potential" of a chemical component of a thermodynamic system: he included kinetic energy ${ }^{10}$, only to show that it must vanish at thermodynamic equilibrium. Guggenheim ${ }^{11}$ treats gravitational energy in the same way as Gibbs, but Lewis and Randall ${ }^{12}$ take the alternative approach and include it in the internal energy. It is unfortunate that few authors except Lewis and Randall ${ }^{12}$ and Denbigh ${ }^{13}$ point out the existing divergences, for there is evidently little prospect of reaching a consensus on this matter in the context of chemical thermodynamics. Considering the thermodynamics of fluid flow (a subject not usually discussed by chemical thermodynamicists) there is much to be said, however, for general adoption of the Landau-Lifshitz approach, because the kinetic and gravitational components of the total energy may often be analysed more or less independently of local thermodynamic parameters. In the absence of uniformity, confusion may be minimized by use of the adjectives "intrinsic" or "total" to indicate the sense in which the terms energy, enthalpy or chemical potential are being used in a particular context.

Department of Earth Sciences,

M. H. Dodson

The University,

Leeds LS2 9JT

Received September 6; revised September 21, 1971.

${ }^{1}$ Waldbaum, D. R., Nature, 232, 545 (1971).

2 Turcotte, D. L., and Oxburgh, E. R., J. Geophys. Res., 74, 1458 (1969).

${ }^{3}$ Pippard, A. B., The Elements of Classical Thermodynamics (Cambridge University Press, 1966).

${ }^{4}$ Liepmann, H. W., and Roshko, A., Elements of Gasdynamics (Wiley, New York, 1958).

${ }^{5}$ Landau, L. D., and Lifshitz, E. M., Fluid Mechanics (Pergamon, London, 1959)

6 Mackenzie, D. P., J. Geophys. Res., 72, 6261 (1967).

7 Tozer, D. C., J. Geomag. Geoelectricity, 22, 35 (1970).

8 Oxburgh, E. R., and Turcotte, D. L., Nature, 218, 1041 (1968).

9 Gibbs, J. W., Collected Works, 1, 144 (Dover, New York, 1961).

10 Gibbs, J. W., Collected Works, 1, 39 (Dover, New York, 1961).

11 Guggenheim, E. A., Thermodynamics (North Holland, Amsterdam, 1959).

12 Lewis, G. N., and Randall, M., Thermodynamics (revised by Pitzer, K. S., and Brewer, L.) (McGraw-Hill, New York, 1961).

13 Denbigh, K. G., Chemical Equilibrium (Cambridge University Press, 1966).

\section{Spontaneous Fission Previously Observed in a Mercury Source}

Previously ${ }^{1}$, we have presented evidence for the possible existence of a superheavy element with atomic number 112 . The principal evidence for the possible existence of this element was based on the observation of spontaneous fission in a mercury source separated from a tungsten target which had been irradiated by $24 \mathrm{GeV}$ protons. Spontaneous fission activity was observed, using polycarbonate foils, in mercury sources separated from two tungsten targets. These latter are identified by the symbols W2 and W3. In the case of the W3 source the proton dose was probably less than for W2 and also only about $30 \%$ of the mercury added as carrier was recovered in the final source (as measured by colorimetric techniques). The observed fission activity was smaller than that observed with the W2 source. In the latter case (W2) the measured activity was originally about three fissions/day and all experimental work reported here has been carried out with this sample.

The argument that the observed activity might be due to a superheavy element was based entirely on the prediction that element 112 will be the chemical homologue of mercury. 